\title{
Medical Comorbidity in a Bipolar Outpatient Clinical Population
}

\author{
John Beyer*,', Maragatha Kuchibhatla', Kenneth Gersing' and K Ranga R Krishnan' \\ 'Department of Psychiatry, Duke University Medical Center, USA
}

\begin{abstract}
The presence of medical illnesses among inpatients with bipolar disorder is known to complicate treatment and lengthen hospital stay. However, except for a few specific diseases, little is known about prevalence of medical illnesses in bipolar outpatients and the effect it may have on treatment. The authors sought to assess the presence of medical illnesses in a large outpatient clinical sample of bipolar patients, and the effect that medical illnesses may have on the clinical assessment and treatment of the underlying bipolar disorder. Using the Duke University Medical Center clinical database, the authors categorized the medical diagnoses of I 379 patients who were treated with bipolar disorder from 2001 to 2002 through outpatient psychiatric clinics. The prevalence of medical comorbidities was examined, as well as the effect their presence had on the clinician's assessment of disease severity and time to improvement. As expected, medical comorbidities increased with age. The most common systemic illnesses in bipolar outpatients were Endocrine and Metabolic Diseases (I3.6\% of the sample), Diseases of the Circulatory System (13.0\%), and Diseases of the Nervous System and Sense Organs (I0.7\%). Significant specific diseases included cardiovascular diseases/hypertension (I0.7\%), COPD/asthma (6.1\%), diabetes (4.3\%), HIV infection (2.8\%), and hepatitis C infection (1.9\%). Clinicians assessed greater severity of illness in patients with increasing numbers of comorbid conditions; however, the time to recovery was not significantly effected by the presence of medical comorbidity. In conclusion, comorbid medical illnesses are common in bipolar outpatients, increasing with age. HIV rates may be increased relative to population norms. Their presence compounds the severity of the illness at time of presentation.

Neuropsychopharmacology (2005) 30, 40I-404, advance online publication, 10 November 2004; doi: I 0. I 038/sj.npp. I 300608
\end{abstract}

Keywords: bipolar; comorbidity; treatment

\section{INTRODUCTION}

The presence of medical comorbidities in patients with bipolar disorder is an anticipated complication of a life-long illness. While it is known that the presence of the comorbidities will effect the course and severity of bipolar disorder and its treatment (American Psychiatry Association, 2002; Black et al, 1998a, b, 1989), few studies have examined which medical comorbidities may commonly be present, or what the impact of these problems may have on patient presentation or course. Further, most studies that have addressed this issue have looked only at bipolar inpatients. This study was undertaken to evaluate how the presence of comorbidities may effect the severity of the illness or time to recovery, and what were the types of medical comorbidities most commonly present in a general outpatient bipolar clinical population. We hypothesized

\footnotetext{
*Correspondence: Dr J Beyer, Box 5319 DUMC, Durham, NC 27710, USA, Tel: + 919668 0209, Fax: +919681 7421,

E-mail: Beyer00I@mc.duke.edu

Received 13 May 2004; revised 13 September 2004; accepted 15 September 2004

Online publication: 6 October 2004 at http://www.acnp.org/citations/ Npp 1006040402 | 8/default.pdf
}

that bipolar patients with more medical comorbidities will have a slower response to treatment that healthier bipolar patients.

\section{METHODS}

\section{Sample}

Since 1998, the Duke University Medical Center (DUMC) Department of Psychiatry has utilized an electronic medical record system in all inpatient wards, outpatient clinics, and emergency rooms. As data is entered, an anonymized longitudinal data repository is built, comprising all qualified visits for each patient. A qualified clinical visit includes information about patient medications, psychiatric diagnosis, side effects, CPT billing codes, Clinician's Global Impression (CGI) scale, and allergies. Information in the data repository is HIPAA compliant and staged for analysis using SAS procedures. Owing to the anonymized design, this study was exempt from IRB review.

The authors queried the DUMC database for all patients diagnosed by their outpatient psychiatrist with Bipolar I disorder regardless of phase. A total of 1379 patients seen through the outpatient clinics from 2001 to 2002 were 
Table I Demographic Information of Sample

\begin{tabular}{|c|c|}
\hline & Value \\
\hline Number & 1379 \\
\hline \multicolumn{2}{|l|}{ Diagnosis } \\
\hline Bipolar, manic/mixed & 603 \\
\hline Bipolar, depressed & 311 \\
\hline Bipolar, unspecified & 69 \\
\hline Bipolar NOS & 396 \\
\hline Mean age (SD) & $32.8(18.3)$ \\
\hline \multicolumn{2}{|l|}{ Gender } \\
\hline Male & 731 (46.9\%) \\
\hline Female & $647(53.0 \%)$ \\
\hline Unknown & I (0.1\%) \\
\hline \multicolumn{2}{|l|}{ Race } \\
\hline White & $895(64.9 \%)$ \\
\hline Black & $236(17.1 \%)$ \\
\hline Hispanic & $8(0.6 \%)$ \\
\hline Asian & $6(0.5 \%)$ \\
\hline Unknown & $76(5 \%)$ \\
\hline
\end{tabular}

identified. Basic demographic measures for the sample were obtained (see Table 1 ). In total, $47 \%$ of the sample was male; $53 \%$ was female. The mean age was 32.8 years $(S D=18.3)$. For comparison of certain disease states, we also identified patients diagnosed with unipolar depression through the DUMC database. Total of 4509 unique patients with unipolar depression were identified. The mean age was 43.2 years $(\mathrm{SD}=9.5)$.

\section{Measurement}

Medical disorders identified by the outpatient psychiatrist as being of significance are recorded in the database. Owing to the large heterogeneity of the medical diagnoses given, the study authors grouped the medical diagnoses into one of the 17 systemic groups based on the classification scheme (International Classification of Diseases, 9th Revision; ICD9) developed by the World Health Organization (1996).

In addition, at each visit, the patient's psychiatrist made an assessment of the overall severity of illness and improvement using the CGI. Utilizing this data, we assessed the effect that the presence of increasing numbers of medical conditions may have on the clinician's assessment of the patient's severity of the illness, and the time to improvement.

\section{Assessment of Method}

The categorization of illnesses into affected systems provides a counting of systemic illnesses and a coarse assessment of medical burden; however, the limitations of this system are self-evident. The medical illnesses are not weighted by severity (thus, Stage 1 and Stage 4 cancer would be given equal weight). Further, using broad categories instead of specific disease identifiers, we decrease the likelihood of identifying potential specific conditions that may be more closely associated with bipolar disorder. It should be noted that clinical information was obtained from active clinical charts. Thus, the medical diagnoses were not recorded or coded by trained medical coding technicians, but rather the patient's doctor, who were trained only in recording illness' presence, rather than codes. In order to compensate for this, several common medical illnesses were identified as being of interest for more specific evaluation, and the prevalence of these was recorded separately.

It should also be noted that all bipolar patients, regardless of their phase at time of first diagnosis, were grouped together for this analysis due to the limited numbers available.

\section{Statistical Analysis}

We used percentages to describe categorical variables, and means and standard deviations to summarize continuous variables. We used multivariate regression to examine the relation between CGI scores of the subject's first visit to the clinic and the number of comorbidities. We used mixed models to analyze the repeated measurements of CGIseverity scores and CGI-improvement scores on the subjects. With this method, each subject's repeated measurements on the dependent variable are first parameterized as an individual growth trajectory plus an error term. In the second stage, the estimated trajectories are modeled as a function of differences between individuals on variables of interest (eg age, number of comorbidities). Age was a covariate in all analyses.

\section{RESULTS}

The demographic data on the 1379 patients with bipolar disorder identified is given in Table 1. The presence of comorbid medical illnesses by category is shown in Table 2. Since several of the ICD-9's systemic categories were infrequently observed in our patient sample (Congenital Anomalies, Conditions Originating in the Perinatal Period, Ill-Defined Conditions, Poisonings), and we were not assessing for psychiatric comorbidities (Mental Disorders), only 13 categories are shown in Table 2 . The prevalence of several medical comorbid conditions that were specifically assessed is shown in Table 3.

In our sample, $56 \%$ did not have any significant comorbid medical conditions; $26 \%$ had one comorbid medical condition, $13 \%$ had two, $3 \%$ had three, and $2 \%$ had four or more comorbid medical conditions. The presence of medical conditions by age of patient is shown in Figure 1.

As expected, the number of comorbid medical conditions increases directly proportional to age. In their 20 s, $30 \%$ of the bipolar patients will have at least one medical condition. This steadily increases throughout life, so that by their $50 \mathrm{~s}$, $50 \%$ of bipolar patients have at least one significant medical condition; and finally in their 70 s, $67 \%$ have at least one significant medical condition.

When the CGI scale scores were analyzed, the number of comorbid conditions was significant in predicting the 
Table 2 Presence of Comorbid Medical Conditions

\begin{tabular}{lcc}
\hline & $\begin{array}{c}\text { Number of } \\
\text { patients }\end{array}$ & $\begin{array}{c}\text { Percent } \\
\text { (\%) }\end{array}$ \\
\hline Infectious and parasitic diseases & 105 & 7.6 \\
Neoplasms & 39 & 2.8 \\
Endocrine, nutritional, and metabolic diseases & 187 & 13.6 \\
Diseases of blood & 21 & 1.5 \\
Diseases of the nervous system and sense organs & 147 & 10.7 \\
Diseases of the circulatory system & 179 & 13.0 \\
Diseases of the respiratory system & 101 & 7.3 \\
Diseases of the digestive system & 101 & 7.3 \\
Diseases of the genitourinary system & 51 & 3.7 \\
Complications of pregnancy, childbirth, and the & 5 & 0.4 \\
puerperi & & \\
Diseases of the skin and subcutaneous tissues & 28 & 2.0 \\
Diseases of the musculoskeletal system and injury & 141 & 10.7 \\
Other & 13 & 0.9 \\
\hline
\end{tabular}

Table 3 Incidence of Specific Medical Illnesses

\begin{tabular}{lcc}
\hline & Number of patients & Percent (\%) \\
\hline Cardiac disease/hypertension & 146 & 10.7 \\
COPD/asthma & 84 & 6.1 \\
Diabetes & 59 & 4.3 \\
HIV & 39 & 2.8 \\
Hepatitis C & 26 & 1.9
\end{tabular}

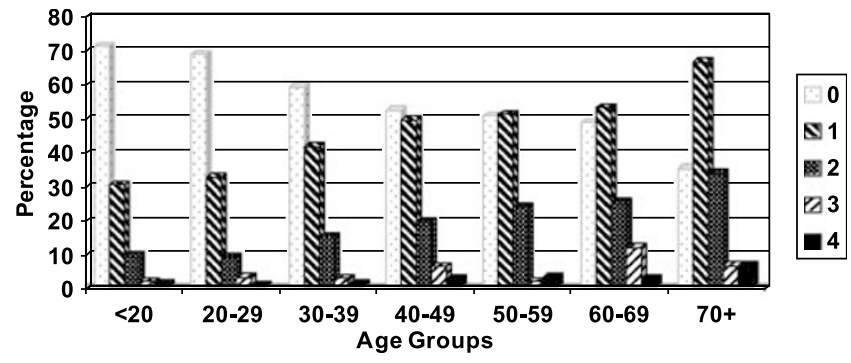

Figure I Percentage of medical comorbidities by age group.

severity of the first visit score $(p=0.03)$. However, the number of comorbidities was not a significant factor in predicting the time to improvement $(p=0.06)$.

\section{DISCUSSION}

To our knowledge, this is the first report assessing medical comorbidities in a psychiatric outpatient clinical population. There have been several previous studies that have examined the presence and response to treatment of specific medical conditions, such as migraines (Mahmood et al,
1999; Fasmer, 2001), vascular risk factors (Cassidy and Carroll, 2002), appendicitis (Ewald et al, 2001), polycystic ovarian disease (McIntyre et al, 2003), and diabetes (Cassidy et al, 1999; Regenold et al, 2002). However, these studies have pursued specific illnesses, rather than look at the broader clinical picture of a psychiatric clinic population.

Black et al (1998a) have retrospectively assessed the impact of medical comorbid illnesses on an inpatient bipolar population. They found that patients with concurrent medical illnesses were associated with greater age, later age of onset, and poorer course of recovery. Strakowski et al (1992), in a prospective study of 60 patients with first episode mania, noted that the presence of a medical comorbidity was associated with an older age, less use of antipsychotic medicine during the hospitalization, shorter hospital stay, and less symptomatology at the time of discharge.

It remains unclear whether bipolar patients are at a higher risk for medical illness than other populations. Strakowski et al (1992) found physical illness in $18 \%$ of their patients with a first episode mania (18\%). However, in a comparative study of bipolar disorder and unipolar depression, Winokur et al (1993) observed that only 3\% of patients with bipolar illness had been physically ill at the time of the diagnosis, a rate much lower than patients with unipolar depression. Adding to the complexity of these findings, Cradock-O'Leary et al (2002) analyzed the use of healthcare systems of mentally ill. They noted that bipolar patients utilize health care services less than other populations though medical burden was similar.

Our findings offer a glimpse of the medical burden that an outpatient clinician may see in their bipolar patients. These data support the hypothesis that the presence of medical comorbidity significantly effects the assessment of severity of a patient's global illness. It also suggests, but does not statistically support the observation that the presence of medical comorbidity lengthens the time to recovery for bipolar outpatients with active disease. In the analyses, these findings were independent of the bipolar patients' age.

Since we did not have a comparison group for the entire model, we were unable to evaluate how the findings compare with other outpatient groups. However, the prevalence of several disease systems deserves comment. The category that was most common was that of Endocrine, Nutritional, and Metabolic Diseases (13.6\% of clinical sample). This included diseases such as diabetes (a condition present in $4.3 \%$ of our population), obesity, thyroid diseases, and hypercholesterolemia among other diagnoses. The rate for diabetes is essentially the same as the national average (4.4\%) (Center for Disease Control 1980-2000), although direct comparison is difficult and could be skewed by the relatively young age of the sample (mean age 32.8 years) or under-reporting by the clinicians.

The second most common category was Diseases of the Circulatory System, affecting 13\% of our clinical sample. In the general population, heart disease is one of the most common medical illnesses. Cardiac disease/hypertension comprised the most common specific illness of our bipolar sample, affecting $10.7 \%$ of the clinical population. 
Diseases of the Nervous System and Sense Organs (11.1\% of the clinical sample) were the third most common category of comorbid medical conditions (10.7\%). This included migraines, head traumas with deficit, stroke, loss of vision, etc.

Finally, an unexpected finding was the Infectious and Parasitic Diseases (7.6\%). Within this category, we found that $2.8 \%$ of our bipolar outpatient sample was diagnosed with HIV. Again, although direct comparison is difficult, this is much higher than anticipated since the national average is $0.3 \%$. Hepatitis C infection was present in $1.9 \%$ of our sample. These findings suggest that bipolar patients may have an increased risk of exposure to life-threatening infection, possibly due to increased risk-taking or impulsive behaviors. Another possible interpretation may be that our sample could be skewed to a more ill outpatient population since the Duke University Medical Center psychiatric clinics are part of a tertiary medical center. We attempted to assess this by querying the same database for the number of unipolar depressed outpatients diagnosed with HIV and hepatitis. The results showed only 46 of 4509 (1.0\%) unipolar depressed outpatients were diagnosed with HIV, and 23 patients $(0.5 \%)$ with hepatitis C. Both of these rates are much lower than that observed in the bipolar population.

Owing to limited studies, the relationship of bipolar illness with comorbid medical diagnoses remains relatively poorly defined. Future research examining rates of medical comorbidity will require a prospective, longitudinal study evaluating bipolar patients and a matched comparison group. The information from such a study would be valuable in anticipating the health risks of our patients, understanding what medical care may be required for our patients, improving access to that care, and understanding the impact a medical illness may have on the course of illness and treatment planning.

\section{ACKNOWLEDGEMENTS}

Support for this research has been provided by NIMH grant \#R01-MH57027 and NARSAD. We also acknowledge Laura Taylor, MS for her assistance with the clinical data information set.

\section{REFERENCES}

American Psychiatry Association (2002). Practice guidelines for the treatment of patients with bipolar disorder (revision). Am J Psychiatry 159(April Suppl): 1-50.

Black DW, Hulbert J, Nasrallah A (1989). The effect of somatic treatment and comorbidity on immediate outcome in manic patients. Compr Psychiatry 30: 74-79.

Black DW, Winokur G, Bell S, Nasrallah A, Hulbert J (1998a). Complicated mania: comorbidity and immediate outcome in the treatment of mania. Arch Gen Psychiatry 45: 232-236.

Black DW, Winokur G, Hulbert J, Nasrallah A (1998b). Predictors of immediate response in the treatment of mania: the importance of comorbidity. Biol Psychiatry 24: 191-198.

Cassidy F, Ahearn E, Carroll J (1999). Elevated frequency of diabetes mellitus in hospitalized manic-depressive patients. Am J Psychiatry 156: 1417-1420.

Cassidy F, Carroll BJ (2002). Vascular risk factors in late onset mania. Psychol Med 32: 359-362.

Center for Disease Control 1980-2000. Diabetes surveillance system: prevalence of diabetes, 1980-2000. www.cdc.gov/diabetes/statistics/prev/national/fig2.htm.

Cradock-O'Leary J, Young AS, Yano EM, Wang M, Lee ML (2002). Use of General medical services by VA patients with psychiatric disorders. Psychiatr Serv 53: 874-878.

Ewald H, Mortensen PB, Mors O (2001). Decreased risk of acute appendicitis in patients with schizophrenia of manic-depressive psychosis. Schiz Res 49: 287-293.

Fasmer OB (2001). The prevalence of migraine in patients with bipolar and unipolar affective disorders. Cephalgia 21: 894-899.

Mahmood T, Romans S, Silverstone T (1999). Prevalence of migraine in bipolar disorder. J Aff Disord 52: 239-241.

McIntyre RS, Mancini DA, McCann S, Srinivasan J, Kennedy SH (2003). Valproate, bipolar disorder and polycystic ovarian syndrome. Bipolar Disord 5: 28-35.

Regenold WT, Thapar RK, Marano C, Gavirneni S, Kondapavuluru PV (2002). Increased prevalence of type 2 diabetes mellitus among psychiatric inpatients with bipolar I and schizoaffective disorders independent of psychotropic drug use. J Aff Disord 70: 19-26.

Strakowski SM, Tohen M, Stoll AL, Faedda GL, Mayer PV, Kolbrener ML et al (1992). Comorbidity in mania at first hospitalization. Am J Psychiatry 149: 554-556.

Winokur G, Coryell W, Endicott J, Akiskal H (1993). Further distinctions between manic-depressive illness and primary depressive disorder. Am J Psychiatry 150: 1176-1181.

World Health Organization (1996). International Classification of Diseases, 9th Revision, Clinical Modification, 5th edn. (ICD-9$\mathrm{CM})$. Practice Management Information Corporation: Los Angeles, CA. 